\title{
Medical image fusion based on variational and nonlinear structure tensor
}

\author{
Xiaobei Wang ${ }^{1, *}$, Rencan $\mathrm{Nie}^{1,2}$, and Xiaopeng Guo ${ }^{1}$ \\ ${ }^{1}$ School of Information Science and Engineering, Yunnan University, Kunming Yunnan, China \\ ${ }^{2}$ School of Automation, Southeast University, Nanjing 210096, China
}

\begin{abstract}
Medical image fusion plays an important role in detection and treatment of disease. Although numerous medical image fusion methods have been proposed, most of them decrease the contrast and lose the image information. In this paper, a novel MRI and CT image fusion method is proposed combining rolling guidance filter, structure tensor, and nonsubsampled shearlet transform (NSST). First, the rolling guidance filter and the sum-modified laplacian (SML) operator are introduced in the algorithm to construct the weight maps in non-linear domain, then the fused gradient is firstly obtained by a new weighted structure tensor fusion method, and the fused image is firstly acquired in NSST domain, finally, a new energy functional is defined to constrain the gradient and pixel information of the final fused image close to the pre-fused gradient and the pre-fused image, experimental results show that the proposed method can retain the edge information of source images effectively and preserve the reduction of contrast.
\end{abstract}

\section{Introduction}

Image fusion is the technique which can combine two or more images into an image, eliminate redundant information and complement unique features between each other. which is widely used in the field of computer vision, the fusion of medical images is an important branch of it. Medical personnel will have more accurate diagnosis after mastering detailed features of human organs. The multi-scales decomposition algorithms are commonly used in medical image fusion, such as discrete wavelet transform (DWT) [1], nonsubsampled contourlet transform (NSCT) [2], and nonsubsampled shearlet transform (NSST) [3]. In these methods, the source images are decomposed into different frequency components, and then different fusion strategies are adopted according to the characteristics of each frequency band, the final result is reconstructed by inverse multi-scales transform. Such approaches can effectively extract the features of the source images. However, they may produce artifacts in the process of image fusion, which results in the reduction of contrast. In order to solve the problems mentioned above, the fusion method based on guided filtering (GFF) is proposed [4], which has achieved remarkable results in eliminating artifacts and preserving the spatial consistency. Based on the idea of the guided filter, an efficient edge-preserving filter named rolling guidance filter was put forward by

\footnotetext{
* Corresponding author: 18314475549@163.com
} 
Zhang Qi et al [5], The method possesses nice properties of bilateral filter and gaussian filter, which can remove the small structure of original images and maintain the sharp image boundary.

Image fusion methods based on variational are proposed in recent years. The boundary, corner and other structural information can be regarded as a special spatial arrangement of gradient information [6]. By adding fusion algorithm into the gradient domain, the fused image can retain the important features of source images efficiently. Considering the advantages of the rolling guidance filter and the gradient domain fusion method, an adaptive image fusion algorithm based on the rolling guidance filter and the structure tensor is proposed in this paper. The flowchart diagram of the proposed method is shown in figure 1 .

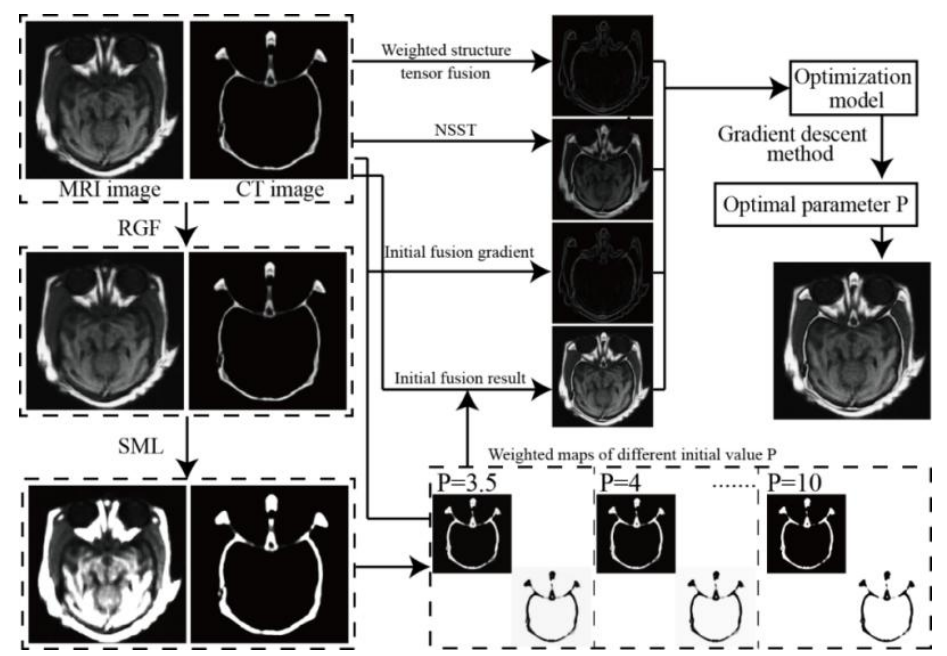

Fig. 1. Diagram of proposed method.

\section{Proposed method}

\subsection{Weighted structure tensor fusion}

The structure tensor is used to represent the gradient information, and the fused structure tensor is firstly obtained in the nonlinear domain. For a set of source images $I_{k}(k=1,2,3 \ldots)$, their structure tensor can be expressed as:

$$
G(x)=\left[\begin{array}{cc}
\sum_{n=1}^{N}\left(\frac{\partial I_{n}}{\partial x_{1}}\right) & \sum_{n=1}^{N} \frac{\partial I_{n}}{\partial x_{1}} \frac{\partial I_{n}}{\partial x_{2}} \\
\sum_{n=1}^{N} \frac{\partial I_{n}}{\partial x_{1}} \frac{\partial I_{n}}{\partial x_{2}} & \sum_{n=1}^{N}\left(\frac{\partial I_{n}}{\partial x_{2}}\right)
\end{array}\right]
$$

Eq1. implies that all the gradients contribute equally to the fused gradient, which results in blurred images and losing of the details of source images.

In order to solve the problems mentioned above, we introduce a new weighted structure tensor fusion, and weight maps are constructed in non-linear domain, which combines the advantages of rolling guidance filter and SML operator, for simplicity, we use RGF to represent the rolling guidance filter, the weight maps can be calculated as:

$$
W_{k}=\frac{e^{p \cdot g_{k}}}{\sum_{k=1}^{N} e^{p \cdot g_{k}}}
$$


where $g_{k}=S_{k} / \sum_{k=1}^{N} S_{k}, S_{k}=S M L\left(r_{k}\right), r_{k}(x)=R G F\left(I_{k}(x)\right)$, and $P$ determines the initial value of the weight map, the weighted structure tensor is obtained by the following formula:

$$
G_{w}(x)=\left[\begin{array}{cc}
\sum_{k=1}^{N}\left(W_{k}(x) \frac{\partial I_{k}}{\partial x_{1}}\right)^{2} & \sum_{k=1}^{N} W_{k}^{2}(x) \frac{\partial I_{k}}{\partial x_{1}} \frac{\partial I_{k}}{\partial x_{2}} \\
\sum_{k=1}^{N} W_{k}^{2}(x) \frac{\partial I_{k}}{\partial x_{1}} \frac{\partial I_{k}}{\partial x_{2}} & \sum_{k=1}^{N}\left(W_{k}(x) \frac{\partial I_{k}}{\partial x_{2}}\right)^{2}
\end{array}\right]
$$

The remainder of the theoretical derivations are given by literature [6], so the fused gradient can be defined as:

$$
V_{F}(x)=\sqrt{\lambda_{1}(x)} \cdot e_{1}(x) \times \operatorname{sign}\left\langle e_{1}(x), \frac{1}{N} \sum_{k=1}^{N} \nabla I_{k}(x)\right\rangle
$$

\subsection{Image fusion based on NSST}

In the optimization model, we need to set a criterion for the pixel level fusion. Considering the shift invariance property of the NSST operator [3], we fuse the source images in the NSST domain. First, the source images $I_{k}$ are decomposed into a low-frequency sub-image $\left\{C_{k}^{l}\right\}$ and a set of high-frequency sub-images $\left\{C_{k}^{l, r}\right\}$, then the average method is employed to combine low-frequency and maximum energy rule is adopted to fuse high-frequency, which can be expressed as follow:

$$
\begin{gathered}
C_{F}^{L}=\frac{1}{N}\left(\sum_{K=1}^{N} C_{k}^{L}\right) \\
C_{F}^{(l, r)}=\max \left(C_{1}^{(l, r)}, C_{2}^{(l, r)} \cdots C_{N}^{(l, r)}\right)
\end{gathered}
$$

Finally, the fused image $C_{F}$ is constructed by inverse nonsubsampled shearlet transform.

\subsection{Optimization model construction}

In order to retain more gradient information and feature information in the fusion result, the energy functional constraints the gradient and the initial fused result close to pre-fused gradient $V_{F}$ and the fusion result $C_{F}$, which can be defined as follow:

$$
E=\arg \min \left\{\left\|\nabla I_{F}{ }^{2}-V_{F}^{2}\right\|^{2}+\beta\left\|I_{F}-C_{F}\right\|^{2}\right\}
$$

Parameter $\beta$ is used to control the balance between the gradient and the fused image, for simplicity: we use $I_{1}, I_{2}$ to denote the source images, and $W_{1}, W_{2}$ to represent the corresponding weight maps, then the gradient descent method is adopted to compute the optimization model:

$$
\begin{gathered}
p_{n+1}=p_{n}+\eta \frac{\partial p}{\partial t} \\
\frac{\partial p}{\partial t}=\frac{d}{d x}\left(\frac{\partial E}{\partial p_{x}}\right)+\frac{d}{d y}\left(\frac{\partial E}{\partial p_{y}}\right)-\frac{\partial E}{\partial p} \\
\frac{\partial E}{\partial p_{x}}=4 \cdot\left(\nabla I_{F}{ }^{2}-\nabla V_{F}^{2}\right) \cdot \frac{\partial W_{1}}{\partial p}\left(I_{1}-I_{2}\right) \cdot f_{x}^{F} \\
\frac{\partial E}{\partial p_{y}}=4 \cdot\left(\nabla I_{F}{ }^{2}-\nabla V_{F}{ }^{2}\right) \cdot \frac{\partial W_{1}}{\partial p}\left(I_{1}-I_{2}\right) \cdot f_{y}^{F} \\
\frac{\partial E}{\partial p}=2 \cdot \beta \cdot\left(I_{F}-C_{F}\right) \cdot \frac{\partial W_{1}}{\partial p}\left(I_{1}-I_{2}\right)
\end{gathered}
$$


where $\nabla I_{F}{ }^{2}=\left(f_{x}^{F}\right)^{2}+\left(f_{y}^{F}\right)^{2}, f_{x}^{F}=\frac{\partial W_{1}}{\partial p} \cdot\left(I_{1}-I_{2}\right) \cdot p_{x}$, and $f_{y}^{F}=\frac{\partial W_{1}}{\partial p} \cdot\left(I_{1}-I_{2}\right) p_{y}$. The optimal valve of $P$ is obtained by the iterative method.

\section{Experimental results}

\subsection{Experimental setup}

We consider five image fusion methods, which include: laplacian pyramid (LAP)-, the discrete wavelet transform (DWT)- [1], nonsubsampled contourlet transform (NSCT)- [2]. NSST- [3], and the guided filter (GF)- [4], based fusion methods for comparison in the experiment. The three objective fusion quality criteria, i.e., an information theory-based metric (MI) [7], an image feature-based metric $\left(Q^{A B / F}\right)$ [8], and universal quality index $\left(Q_{0}\right)$ [9] are adopted to assess the fusion results.

The iteration number of RGF is set to $4, \delta_{s}$ is 1.2 and $\delta_{r}$ is 0.05 . The decomposition level of DWT and LAP is 4. Average coefficient is adopted for low frequency sub-images, and absolute maximum choosing scheme is used to fuse high-frequency coefficients for DWT, LAP, NSCT and NSST. Four decomposition levels with 2, 8, 8, 16 directions from coarser scale to finer scale are adopted for NSCT and NSST methods. The parameter for GF-based fusion method is given by the author [4].

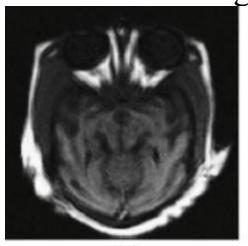

(a)

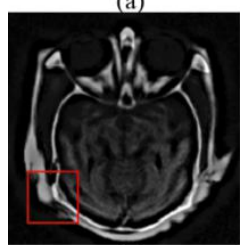

(e)

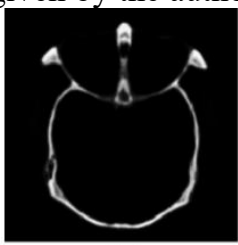

(b)

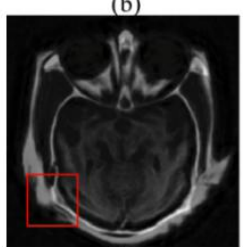

(f)

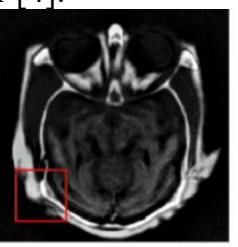

(c)

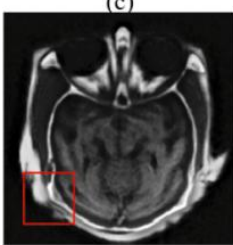

(g)

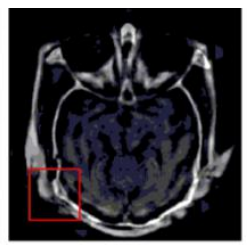

(d)

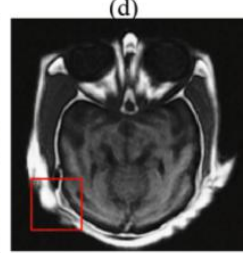

(h)

Fig. 2. Fusion results using "Med-1", i.e., (a) MRI image; (b) CT image; (c) LAP; (d) DWT; (e) NSCT; (f) NSST; (g) GFF; (h) Proposed method.

\subsection{Experimental results and discussion}

Figure 2 show the fusion results obtained with different methods for "Med-1", figure 2(d) shows the result of DWT-based method consists of some obvious artifacts, figure 2(c) and figure $2(\mathrm{~g})$ are acquired by LAP- and GF-base method, respectively, which show fewer artifacts, but contrast has been decreased, figure 2(e) shows the result of NSCT-base method, we can see some details are missed in the process of fusion. Figure 2(f) is obtained by NSST-based method, which also has the problem of contrast reduction. The proposed method has a better performance in visual effect, which retains more edge information and details, and enhance the contrast. Another experiment is shown in figure 3, as we can see from the marked region, some details of source images are lost, but our method preserve the details effectively. We don't show the three addition fusion results, they are similar to previous results. 


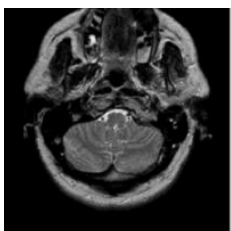

(a)

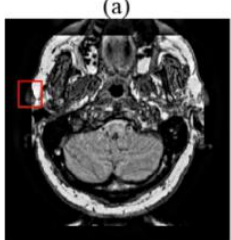

(e)

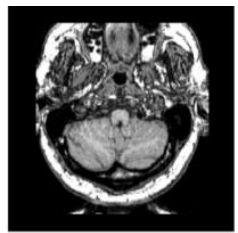

(b)

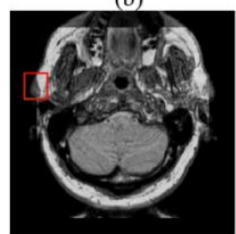

(f)

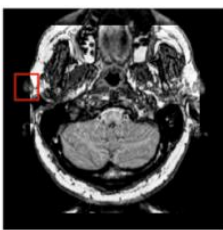

(c)

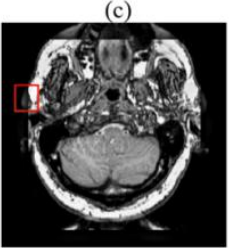

(g)

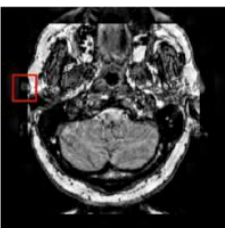

(d)

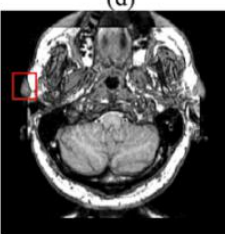

(h)

Fig. 3. Fusion results using "Med-2", i.e., (a) MRI image; (b) CT image; (c) LAP; (d) DWT; (e) NSCT; (f) NSST; (g) GFF; (h) Proposed method.

Quantitative comparison of different fusion methods' performance for five test images are given in table 1. It is obvious that the proposed method outperforms the other methods in all object criteria. The highest MI illustrates the proposed method obtain more details than other methods, highest $Q^{A B / F}$ show that more edge information is transferred tos the fusion result, and highest $Q_{0}$ represents our result is more similar to the source images.

Table 1. Objective criteria of different methods for the test image sets.

\begin{tabular}{|c|c|c|c|c|}
\hline Test images & method & MI & $Q^{A B / F}$ & $Q_{0}$ \\
\hline \multirow{6}{*}{ Med-1 } & LAP & 1.292 & 0.746 & 0.586 \\
\hline & DWT & 1.044 & 0.627 & 0.583 \\
\hline & NSCT & 1.104 & 0.704 & 0.617 \\
\hline & NSST & 1.478 & 0.474 & 0.553 \\
\hline & GFF & 1.725 & 0.790 & 0.859 \\
\hline & Proposed method & 3.250 & 0.817 & 0.937 \\
\hline \multirow{6}{*}{ Med-2 } & LAP & 1.703 & 0.498 & 0.385 \\
\hline & DWT & 1.576 & 0.449 & 0.355 \\
\hline & NSCT & 1.643 & 0.492 & 0.388 \\
\hline & NSST & 1.784 & 0.377 & 0.363 \\
\hline & GFF & 1.834 & 0.572 & 0.383 \\
\hline & Proposed method & 2.994 & 0.636 & 0.418 \\
\hline \multirow{6}{*}{ Med-3 } & LAP & 1.680 & 0.557 & 0.442 \\
\hline & DWT & 1.522 & 0.511 & 0.442 \\
\hline & NSCT & 1.617 & 0.564 & 0.467 \\
\hline & NSST & 1.838 & 0.447 & 0.468 \\
\hline & GFF & 2.042 & 0.654 & 0.492 \\
\hline & Proposed method & 2.948 & 0.661 & 0.536 \\
\hline \multirow{6}{*}{ Med-4 } & LAP & 1.695 & 0.533 & 0.364 \\
\hline & DWT & 1.590 & 0.479 & 0.336 \\
\hline & NSCT & 1.640 & 0.532 & 0.364 \\
\hline & NSST & 1.769 & 0.425 & 0.341 \\
\hline & GFF & 1.973 & 0.614 & 0.366 \\
\hline & Proposed method & 2.775 & 0.641 & 0.384 \\
\hline
\end{tabular}




\begin{tabular}{|c|c|c|c|c|}
\hline \multirow{4}{*}{ Med-5 } & LAP & 1.642 & 0.536 & 0.413 \\
\cline { 2 - 5 } & DWT & 1.501 & 0.484 & 0.401 \\
\cline { 2 - 5 } & NSCT & 1.580 & 0.538 & 0.428 \\
\cline { 2 - 5 } & NSST & 1.786 & 0.444 & 0.438 \\
\cline { 2 - 5 } & GFF & 1.869 & 0.621 & 0.454 \\
\cline { 2 - 5 } & Proposed method & $\mathbf{2 . 8 2 0}$ & $\mathbf{0 . 6 4 8}$ & $\mathbf{0 . 5 0 2}$ \\
\hline
\end{tabular}

\section{Conclusion}

In this paper, a novel image fusion algorithm for MRI and CT images is proposed, we take advantage of rolling guidance filter and SML operator to construct weight maps in nonlinear domain, then obtain the fused gradient by weighted structure tensor and fused image in NSST domain, finally, a new optimization model is built to get the final fused result, experimental results show the proposed method has a good performance in both visual effects and object evaluation.

\section{Acknowledgments}

This research was financially supported by the National Natural Science Foundation of China (nos. 61463052 and 61365001).

\section{References}

1. Rajarshi K, Himabindu C. DWT based medical image fusion with maximum local extrema[C]//Computer Communication and Informatics (ICCCI), 2016 International Conference on. IEEE, 2016: 1-5.

2. Li T, Wang Y. Biological image fusion using a NSCT based variable-weight method[J]. Information Fusion, 2011, 12(2): 85-92.

3. Yin M, Liu W, Zhao X, et al. A novel image fusion algorithm based on nonsubsampled shearlet transform[J]. Optik-international journal for light and electron optics, 2014, 125(10): 2274-2282.

4. Li S, Kang X, Hu J. Image fusion with guided filtering[J]. IEEE Transactions on Image Processing, 2013, 22(7): 2864-2875.

5. Zhang $\mathrm{Q}$, Shen $\mathrm{X}, \mathrm{Xu} \mathrm{L}$, et al. Rolling guidance filter[C]//European Conference on Computer Vision. Springer, Cham, 2014: 815-830.

6. Liu X, Mei W, Du H. Structure tensor and nonsubsampled shearlet transform based algorithm for CT and MRI image fusion[J]. Neurocomputing, 2017, 235: 131-139.

7. Yang B, Li S. Pixel-level image fusion with simultaneous orthogonal matching pursuit[J]. Information fusion, 2012, 13(1): 10-19.

8. Xydeas C S, Petrovic V. Objective image fusion performance measure[J]. Electronics letters, 2000, 36 (4): 308-309.

9. Piella $\mathrm{G}$, Heijmans H. A new quality metric for image fusion[C]//Image Processing, 2003. ICIP 2003. Proceedings. 2003 International Conference on. IEEE, 2003, 3: III173. 